\title{
Analysis of Heat Transfer Influence Factors in the Equipment System Xin REN ${ }^{1,2}$, Li MA ${ }^{2 *}$
}

1. Graduate School, National Defense University, Beijing, 100091, China

2. Medical Protection Laboratory, Naval Medical Research Institute, Shanghai, 200433, China

*Corresponding author

Key words: equipment system; additional acceleration; flow path; thermal conductivity model Abstract: In this paper, the transient model of heat transfer is established based on the two fluid model and the non-steady state heat conduction model of solid wall. The additional acceleration model is introduced in order to study the influence of flow channel shape and motion on the boiling flow and heat transfer in the runner. The results of the research have guiding significance for the design and application of the relevant core flow channel.

\section{Introduction}

In this paper, the transient two-phase flow and heat transfer model is established based on two fluid model and relevant numerical method and additional acceleration model is introduced in oeder to predict and reveal the heat transfer characteristics and the influence rules of the runner under the condition of the shape and movement, and it can provide the basis for the experimental design and relevant application.

\section{Analysis model}

In this paper, the two fluid model is used to simulate the flow and heat transfer behavior of the fluidized bed. The assumptions of the two phase flow and heat transfer model mainly include as follows:

(1)the vapor and liquid phase parameters are changed only along the flow direction of $\mathrm{z}$.

(2)the vapor and liquid phases are incompressible.

(3) the energy dissipation due to Reynolds stress is neglected, etal.

The related equations are as follows:

(1) vapor and liquid phase mass equation:

$$
\frac{\partial\left(\alpha_{k} \rho_{k}\right)}{\partial t}+\frac{\partial\left(\alpha_{k} \rho_{k} v_{k}\right)}{\partial z}=\Gamma_{k}
$$

(2) momentum equation of vapor and liquid phase:

$$
\frac{\partial\left(\alpha_{k} \rho_{k} v_{k}\right)}{\partial t}+\frac{\partial\left(\alpha_{k} \rho_{k} v_{k}^{2}\right)}{\partial z}=\Gamma_{k} v_{\mathrm{j}, k}-\alpha_{k} \frac{\partial p}{\partial z}-g \alpha_{k} \rho_{k}-\left(\frac{\partial p}{\partial z}\right)_{\mathrm{w}, k}-\left(\frac{\partial p}{\partial z}\right)_{\mathrm{i}, k}
$$

(3) energy equation of vapor and liquid phase:

$$
\alpha_{k} \rho_{k} \frac{\partial\left(h_{k}\right)}{\partial t}+\alpha_{k} \rho_{k} \frac{\partial\left(v_{k} h_{k}\right)}{\partial z}=\alpha_{k} \frac{\partial p}{\partial t}+\alpha_{k} v_{k} \frac{\partial p}{\partial z}+Q_{\mathrm{w}, k}+Q_{\mathrm{i}, k}+\Gamma_{k} h_{\mathrm{j}, k}^{\prime}
$$

In the formula, $\alpha, \rho, v, h, \Gamma$ is respectively void fraction, density, velocity, enthalpy and phase mass transfer rate. If subscript $\mathrm{k}$ is l,it is expressed as liquid phase, and if subscript $\mathrm{k}$ is g,it is expressed as vapor. $\left(\frac{\partial p}{\partial z}\right)_{\mathrm{w}, k},\left(\frac{\partial p}{\partial z}\right)_{\mathrm{i}, k}$ is respectively the pressure drop source term of $\mathrm{k}$ phase wall friction and interfacial friction. $Q_{\mathrm{w}, k}, Q_{\mathrm{i}, k}$ is respectively heat transfer item of k phase wall and interphase. The source term relationship and the boiling point are all calculated with the relevant experence relationship.

The equations of solid wall side unsteady heat conduction model (two dimensional) are as follows: 


$$
\rho_{\mathrm{w}} c_{p, \mathrm{w}} \frac{\partial T_{\mathrm{w}}}{\partial t}=\frac{\partial}{\partial x}\left(\lambda_{\mathrm{w}} \frac{\partial T_{\mathrm{w}}}{\partial x}\right)+\frac{\partial}{\partial z}\left(\lambda_{\mathrm{w}} \frac{\partial T_{\mathrm{w}}}{\partial z}\right)+\dot{S}
$$

In the formula, $c_{p}, \lambda$ is respectively the coefficient of specific heat and thermal conductivity, $\mathrm{T}_{\mathrm{w}}$ is the wall temperature, $x$ is the wall thickness direction, and $\dot{S}$ is the internal heat source.

\section{Case analysis}

3.1 Simulation results

The comparison between the calculated results of wall heat transfer coefficient and the measured results under typical operating conditions is shown in figure 1, and it can be seen that the calculated values and the experimental values fit very well.

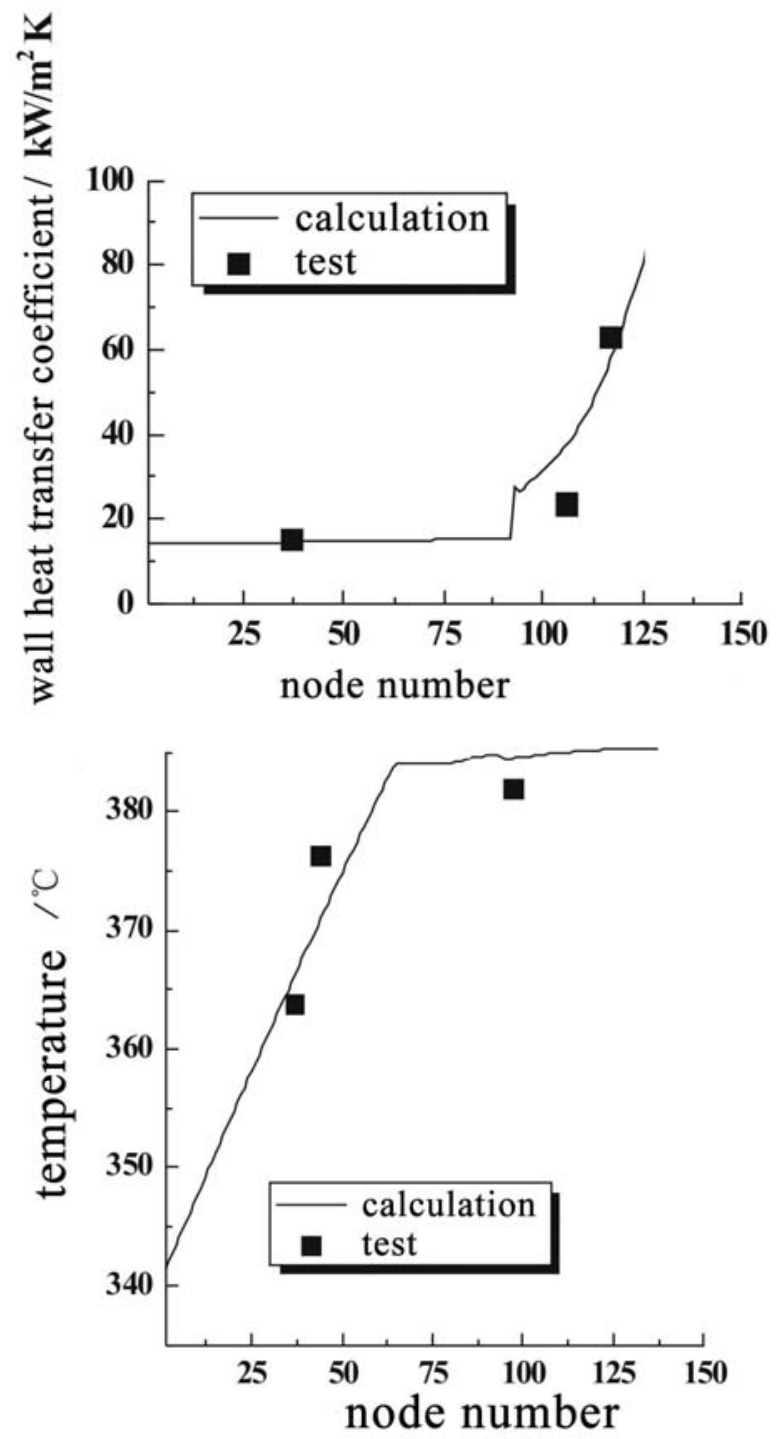

Fig. 1 Comparison of calculated wall heat transfer coefficient and wall temperature with test data

\subsection{The effect of two phase boiling heat transfer}

The distribution of the pressure along the channel, the wall heat transfer coefficient and the wall temperature and the temperature in the center of the nuclear power plant core flow channel can be shown in figure 2 4. It can be seen that the pressure is affected greatly in different angle of inclination, the boiling heat transfer in the flow channel and the wall temperature are less affected by the spatial orientation. 


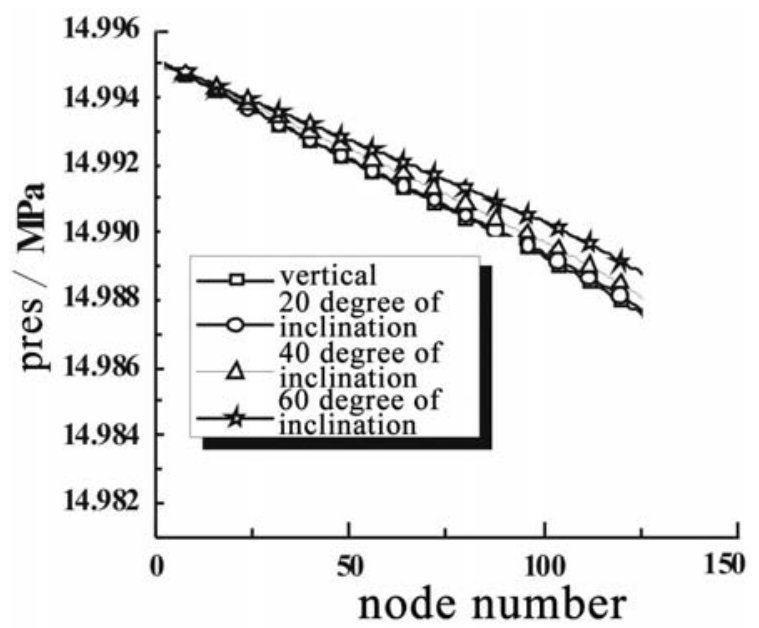

Fig.2 Distribution of the pressure in different angle of inclination

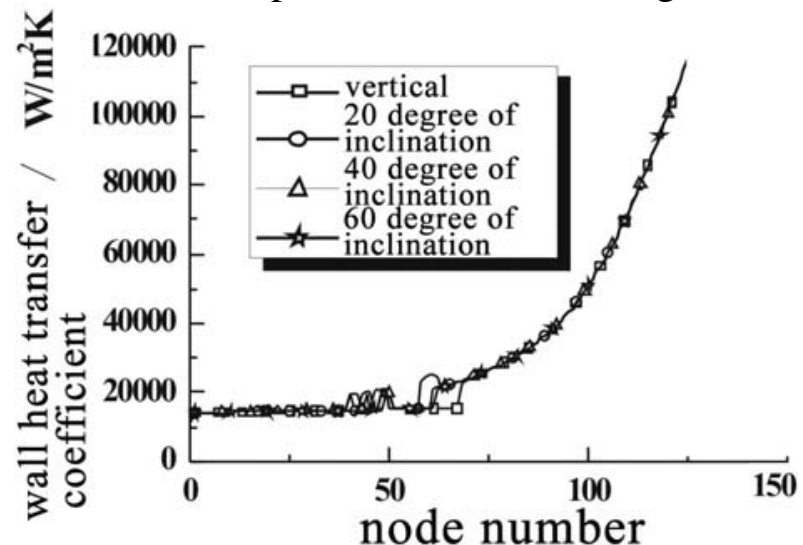

Fig.3 Distribution of the wall heat transfer coefficient in different angle of inclination
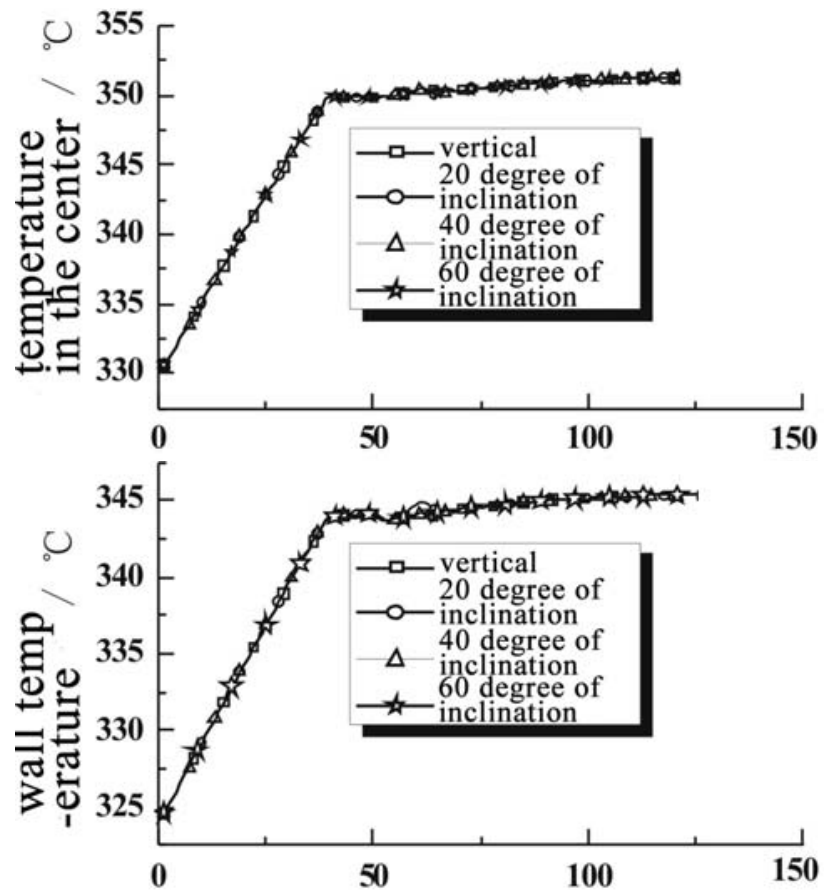

Fig.4 Distribution of the wall temperature and the temperature in the center in different angle of inclination

\section{Conclusion}

The fluctuation of flow channel can cause the following fluctuation of corresponding parameter. 
The pressure fluctuation is slightly obvious, and pulse amplitude of the rest heat transfer parameters is small. The fluctuations of the parameters increase with the swing acceleration, the swing frequency had little effect on the fluctuations of the parameters, and the mean of parameters are basically unchanged under the conditions of movement and static condition.

\section{References}

[1] Wenquan Tao. Numerical heat transfer theory[M].Xi'an: Xi'an Jiao Tong University press, 2001.

[2] Kunli Wen, Yifang Huang,You Mei-li,et al.The development of GM(1,1)[J].IEEE International Conference on Intelligent and Advanced Systems,pp.276-279,2007.

[3] Julong Deng. Grey prediction and decision [M]. Wuhan: Huazhong university of science and technology press, 1986.

[4] Jizhou GUO, Guibao SONG. PENG Shao-xiong. Analysis for operational and support cost of equipment using grey model[J]. Systems Engineering and Electronics, 26(1),pp. 64-67,2004.

[5]Deng J L. Control problems of grey systems[J].System\&Control Letters, 1(5),pp.288-294,1982.

[6]Gunnar Johanson,Jan Holmberg,Safety evaluation by living probability safety assessment:procedures and applications for planning of operational activities and analysis of operating experience.SKI Report94:2,Swedish Nuclear Power Inspectorate,Stockholm,1994.

[7]International Atomic Energy Agency. Component Reliability Data for Use in Probabilistic Safety Assessment[R]. IAEA-TECDOC-478, 1988.

[8] Guangchen Bai, Yan Chen. Satisfactory solution of multi-objective optimization of system reliability[J]. Systems engineering and electronics, 22(8),pp.85-87,2000. 